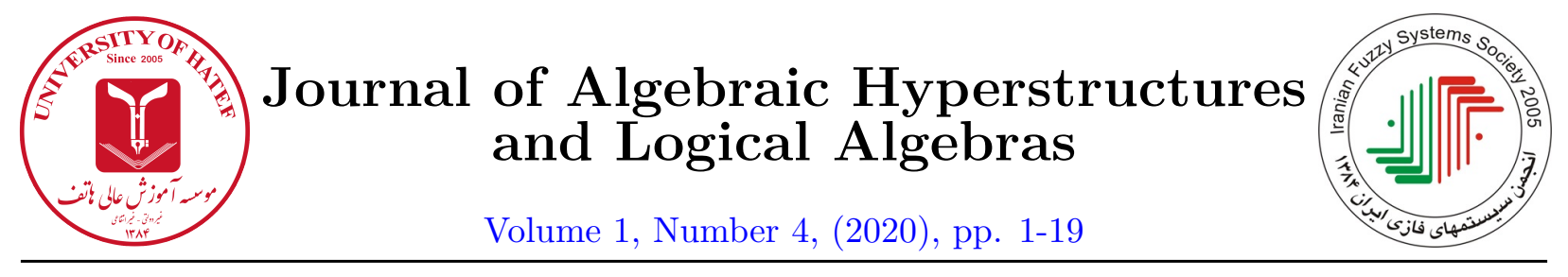

\title{
Lattices of fractions and flat morphisms of bounded distributive lattices
}

\author{
G. Georgescu ${ }^{1}$ \\ ${ }^{1}$ University of Bucharest, Faculty of Mathematics and Computer Science, Bucharest, Romania
}

georgescu.capreni@yahoo.com

\begin{abstract}
The lattices of fractions were introduced by Brezuleanu and Diaconescu in 1969. They used this concept in order to construct a Grothendieck - style duality for the category $\mathbf{D}_{01}$ of bounded distributive lattices. Then the lattices of fractions are studied in connection with other themes in lattice theory: lattices schemas, localization of bounded distributive lattices, sheaf representations of normal lattices,etc.

This paper continues this research vein. We relate the lattices of fractions to flat lattice morphisms, patch and flat topologies on the spectra of bounded distributive lattices, conormal and Stone lattices, etc.

We define the flat morphisms of $\mathbf{D}_{01}$ in terms of the residuation operation existing in the frames of lattice ideals. We study how the lattices of fractions preserve the flatness property of morphisms. Two characterization theorems of flat and patch topologies are proved. The lattices of fractions are used for obtaining new characterizations of conormal and Stone lattices.
\end{abstract}

\section{Introduction}

The lattices of fractions were introduced by Brezuleanu and Diaconescu in [10] in order to obtain a Grothendieck-like duality for the category $\mathbf{D}_{01}$ of bounded distributive lattices. They are the lattice-theoretic version of rings of fractions [4] and there exists much similarity between their theories. The subject was developed in relationship with other themes: Lattices schemas [9], localization theory of bounded distributive lattices [19], [20], sheaf representations of normal lattices [22], etc.

https://doi.org/10.29252/HATEF.JAHLA.1.4.1 
The aim of this paper is to use the lattices of fractions in the study of flat morphisms of $\mathbf{D}_{01}$, flat and patch topologies on the spectra of a bounded distributive lattice, as well as to obtain new characterizations of conormal and Stone lattices.

The notion of flat ring morphism plays an important role in commutative algebra and algebraic geometry [4], [8], [15], [1]. Usually, the flat ring morphisms are defined by using the tensor product of commutative rings (see eg. [4], p.30).

By Exercise 22 of [8], p.65 or [32], p.46, a morphism $f: R \rightarrow Q$ of commutative rings is flat if and only if $(I: J) Q=(I Q: J Q)$, where $I, J$ are ideals of $R$ and $J$ is finitely generated. We remark that this characterization of flat ring morphisms is expressed in terms of residuation operation in lattices of ring ideals. This observation is the starting point in order to define the flat morphisms in the category $\mathbf{D}_{01}$.

If $A \in \mathbf{D}_{01}$, then the set $\operatorname{Id}(A)$ of ideals in $A$ is a frame [27], hence $\operatorname{Id}(A)$ is endowed with a residuation operation. Recall that if $I$ is an ideal of $A$ and $a \in A$, then $(I: a)=\{x \in A \mid x \wedge a \in I\}$ is an ideal of $A$. Each morphism $u: A \rightarrow B$ of $\mathbf{D}_{01}$ induces a map $u^{\bullet}: \operatorname{Id}(A) \rightarrow \operatorname{Id}(B)$ : For each $I \in I d(A), u^{\bullet}(I)$ is the ideal $(u(I)]$ of $B$ generated by $u(I)$.

A morphism $u: A \rightarrow B$ of $\mathbf{D}_{01}$ is said to be flat if $u^{\bullet}(I: x)=\left(u^{\bullet}(I): u(x)\right)$, for all $I \in I d(A)$ and $x \in A$.

The first goal of this paper is to connect the lattices of fractions and the flat morphisms of bounded distributive lattices. In particular, we emphasize how the flateness of lattice morphisms is preserved by lattices of fractions construction. Our second goal is to characterize the flat topology on prime spectra of a bounded distributive lattice in terms of lattices of fractions. Besides them, by using some lattices of fractions we obtain new characterizations of conormal and Stone lattices.

The paper is organized as follows: Section 2 contains two theorems on the prime spectra of colimits in $\mathbf{D}_{01}$. In Section 3, we recall from [10] the definition of lattice of fractions $L / S$ associated with a $\wedge$-closed subset $S$ of bounded distributive lattice $L$, as well as some basic properties of prime and minimal prime spectra of $L / S$.

Section 4 concerns the flat morphisms in $\mathbf{D}_{01}$. Similar to the case of rings [4], [8], the main example of flat morphism in $\mathbf{D}_{01}$ is the canonical morphism $\pi_{S}: L \rightarrow L / S$ associated with a $\wedge$-closed subset $S$ of $L$. Some characterization theorems of flat lattice morphisms are proved. We show that the flatness property is preserved by the lattice of fractions and colimit constructions.

In Section 5, we obtain an algebraic characterization of flat topology on the prime spectrum $\operatorname{Spec}(L)$ in terms of lattices of fractions. Another result describes the patch topology on $\operatorname{Spec}(L)$. These two theorems (= Theorems 5.4 and 5.10) can be view as a lattice counterpart of some results of Tarizadeh [36] on the flat and patch topologies on the prime spectra of rings. Similar to the rings case [36], much of the flat and patch topologies theory can be developed by using Theorems 5.4 and 5.10. Two theorems of Section 6 characterize conormal and Stone lattice in terms of some lattices of fractions.

\section{Preliminaries}

Let $L$ be a bounded distributive lattice. Then $\operatorname{Id}(L)$ the frame of its ideals [27] and $\operatorname{Spec}(L)$ the set of prime ideals of $L$. $\operatorname{Spec}(L)$ is called the prime spectrum of $L$. For any ideal $I$ of $L$ we denote $D(I)=\{P \in \operatorname{Spec}(L) \mid I \nsubseteq P\}$ and $V(I)=\{P \in \operatorname{Spec}(L) \mid I \subseteq P\}$. In particular, if $a \in L$, then we denote $D(a)=\{P \in \operatorname{Spec}(L) \mid a \notin P\}$ and $V(a)=\{P \in \operatorname{Spec}(L) \mid a \in P\}$. According to [5], [27], $(D(I))_{I \in I d(L)}$ is the family of open sets for the Stone topology on $\operatorname{Spec}(L)$. The family $(D(a))_{a \in L}$ is a basis of compact open sets for this topology. The set $\operatorname{Max}(L)$ of maximal ideals in $L$ is called the maximal spectrum of $L$. Then $\operatorname{Max}(L) \subseteq \operatorname{Spec}(L)$ and $\operatorname{Max}(L)$ is a subspace of 
$\operatorname{Spec}(L)$ w.r.t. the Stone topology.

Following [5], $\mathbf{D}_{01}$ will denote the category of bounded distributive lattices. Any morphism $f: L \rightarrow L^{\prime}$ of $\mathbf{D}_{01}$ induces a map $f^{*}: I d\left(L^{\prime}\right) \rightarrow I d(L)$ defined by $f^{*}(J)=f^{-1}(J)$, for all $J \in I d\left(L^{\prime}\right)$. By restricting $f^{*}$ to prime ideals one obtains a continuous map $f^{*}: \operatorname{Spec}\left(L^{\prime}\right) \rightarrow \operatorname{Spec}(L)$.

We shall recall from [5] the construction of direct inductive limits (= colimits) in the category $\mathbf{D}_{01}$. Let us consider an inductive system $\left(L_{i}, u_{i j}: L_{i} \rightarrow L_{j}\right)_{i \leq j}$ in $\mathbf{D}_{01}$, indexed by the direct ordered set $(I, \leq)$. We take the following equivalence relation $\sim$ on the disjoint union $\bigsqcup_{i \in I} L_{i}$ : For all $x \in L_{i}$ and $y \in L_{j}, x \sim y$ if and only if there exists an element $k$ of $I$ such that $i \leq k$, $j \leq k$ and $u_{i k}(x)=u_{j k}(y)$. Then $\operatorname{colim}_{i \in I} L_{i}=\bigsqcup_{i \in I} L_{i} / \sim$ is a bounded distributive lattice, named the colimit of the system $\left(L_{i}, u_{i j}: L_{i} \rightarrow L_{j}\right)_{i \leq j}$ in $\mathbf{D}_{01}$. For any $x \in \bigsqcup_{i \in I} L_{i}, x / \sim$ will denote the equivalence class of $x$. Let us consider the canonical morphisms $u_{i}: L_{i} \rightarrow \operatorname{colim}_{i \in I} L_{i}$, $i \in I$, defined by $u_{i}(x)=x / \sim$, for all $x \in L_{i}$. The colimit colim $m_{i \in I} L_{i}$ has the following property of universality: For any family $\left(f_{i}: L_{i} \rightarrow A\right)_{i \in I}$ of morphisms in $\mathbf{D}_{01}$ such that $f_{i} \circ u_{i j}=f_{j}$, for all $i \leq j$, there exists a unique morphism $f: \operatorname{colim}_{i \in I} L_{i} \rightarrow A$ such that $f \circ u_{i}=f_{i}$, for all $i \in I$.

Keeping the previous notations we shall present some known results on the prime spectrum $\operatorname{Spec}\left(\operatorname{colim}_{i \in I} L_{i}\right)$. For sake of completeness we shall sketch their proofs.

Lemma 2.1. For any $P \in \operatorname{Spec}\left(\operatorname{colim}_{i \in I} L_{i}\right)$ we have $P=\bigcup_{i \in I} u_{i}^{-1}(P) / \sim$.

For any $i \in I$, consider the continuous map $u_{i}^{*}: \operatorname{Spec}\left(\operatorname{colim}_{i \in I} L_{i}\right) \rightarrow \operatorname{Spec}\left(L_{i}\right)$ induced by the lattice morphism $u_{i}: L_{i} \rightarrow \operatorname{colim}_{i \in I} L_{i}$.

Proposition 2.2. $\operatorname{Spec}\left(\operatorname{colim}_{i \in I} L_{i}\right)=\bigcap_{i \in I} u_{i}^{*}\left(\operatorname{Spec}\left(L_{i}\right)\right)$.

Proof. We shall prove that for each ideal $P$ of $\operatorname{colim}_{i \in I} L_{i}$, the following equivalence holds:

$P \in \operatorname{Spec}\left(\operatorname{colim}_{i \in I} L_{i}\right)$ if and only if $u_{i}^{-1}(P) \in \operatorname{Spec}\left(L_{i}\right)$, for all $i \in I$.

The implication $(\Rightarrow)$ is obvious. In order to prove that the converse implication $(\Leftarrow)$, assume that $u_{i}^{-1}(P) \in \operatorname{Spec}\left(L_{i}\right)$, for all $i \in I$. Let $a, b$ be two elements of $\operatorname{colim}_{i \in I} L_{i}$ such that $a \wedge b \in P$, hence there exist $i, j \in I$ such that $x \in L_{i}, y \in L_{j}, a=x / \sim$ and $b=y / \sim$. Thus $a \wedge b=x / \sim \wedge y / \sim$ $=u_{k}\left(u_{i k}(x) \wedge u_{j k}(y)\right)$, hence $u_{i k}(x) \wedge u_{j k}(y) \in u_{k}^{-1}(P)$. Since $u_{k}^{-1}(P)$ is a prime ideal of $L_{k}$, it follows that $u_{i k}(x) \in u_{k}^{-1}(P)$ or $u_{j k}(y) \in u_{k}^{-1}(P)$, so $a=u_{k}\left(u_{i k}(x)\right) \in P$ or $b=u_{k}\left(u_{j k}(y)\right) \in P$. Therefore $P$ is a prime ideal of $\operatorname{colim}_{i \in I} L_{i}$.

Proposition 2.3. $f^{*}\left(\operatorname{Spec}\left(\operatorname{colim}_{i \in I} L_{i}\right)\right)=\bigcap_{i \in I}\left(f_{i}^{*}\left(\operatorname{Spec}\left(L_{i}\right)\right)\right.$.

Proof. The map $f^{*}$ preserves the arbitrary intersections, hence by using Proposition 2.2 the following equalities hold:

$$
f^{*}\left(\operatorname{Spec}\left(\operatorname{colim}_{i \in I} L_{i}\right)\right)=f^{*}\left(\bigcap_{i \in I} u_{i}^{*}\left(\operatorname{Spec}\left(L_{i}\right)\right)\right)=\bigcap_{i \in I} f^{*}\left(u_{i}^{*}\left(\operatorname{Spec}\left(L_{i}\right)\right)\right)=\bigcap_{i \in I}\left(f_{i}^{*}\left(\operatorname{Spec}\left(L_{i}\right)\right) .\right.
$$

\section{Lattices of fractions}

The lattices of fractions were introduced by Brezuleanu and Diaconescu in [10]. In this section we shall recall some basic facts on the lattices of fractions and we give new proofs for some known properties of prime ideals.

Let us fix a bounded distributive lattice $L$. A subset $S$ of $L$ is $\wedge$-closed if $1 \in S$ and $x, y \in S$ implies $x \wedge y \in S$. For any $\wedge$-closed subset $S$ of $L$ consider the following congruence relation: $x \equiv_{S} y$ if and only if there exists $t \in S$ such that $x \wedge t=y \wedge t$. Following [10], the quotient lattice 
$L / S=L / \equiv_{S}$ is called the lattice of fractions of $L$ modulo $\equiv_{S}$. We remark that any filter of $L$ is a $\wedge$-closed subset of $L$. If $[S)$ is the filter generated by a $\wedge$-closed subset $S$ of $L$, then $\equiv_{S}=\equiv_{[S}$, hence $L / S=L /[S)$. For any element $a \in L, a / S$ will denote the congruence class of $a$ modulo $\equiv_{S}$.

Let us consider the surjective morphism $\pi_{S}: L \rightarrow L / S$, defined by $\pi_{S}(x)=s / S$, for each $x \in L$. Thus $x / S=1 / S$ if and only if $x \in[S)$ and $L / S$ is a nontrivial lattice if and only if $0 \notin[S)$. Following Proposition 1.4 of [10], recall the universality property of the lattice of fractions $L / S$ : For each morphism $f: L \rightarrow A$ such that $f(S)=1$ there exists a unique morphism $g: L / S \rightarrow A$ such that $g \circ \pi_{S}=f$.

Lemma 3.1. $\quad$ (1) If $I \in I d(L)$, then $\pi_{S}(I)$ is an ideal of $L / S$;

(2) For any ideal $J$ of $L / S$ we have $\pi_{S}\left(\pi_{S}^{-1}(J)\right)=J$.

If $\pi_{s}^{*}: \operatorname{Spec}(L / S) \rightarrow \operatorname{Spec}(L)$ is the continuous map associated with $\pi_{s}^{*}$, then $\operatorname{Im}\left(\pi_{s}^{*}\right)=$ $\{P \in \operatorname{Spec}(A) \mid P \cap S=\emptyset\}$ (see Observation 1.1 of [10]).

Lemma 3.2. 10] $\pi_{s}^{*}: \operatorname{Spec}(L / S) \rightarrow\{P \in \operatorname{Spec}(L) \mid P \bigcap S=\emptyset\}$ is a homeomorphism.

We observe that the map $\pi_{s}^{*}: \operatorname{Spec}(L / S) \rightarrow\{P \in \operatorname{Spec}(L) \mid P \bigcap S=\emptyset\}$ is an order-isomorphism.

Let $(I, \leq)$ be a directed ordered set and $\left(S_{i}\right)_{i \in I}$ be a family of $\wedge$-closed subsets of $L$ such that $S_{i} \subseteq S_{j}$ for all $i \leq j$ in $I$. For any pair $(i, j)$ such that $i \leq j$ we denote $u_{i j}: L / S_{i} \rightarrow L / S_{j}$ the lattice morphism defined by $u_{i j}\left(x / S_{i}\right)=x / S_{j}$, for all $x \in L$.

Lemma 3.3. $[10]$

(1) $\left(L / S_{i}, u_{i j}\right)_{i \leq j}$ is an inductive system in $\mathbf{D}_{01}$;

(2) $S=\bigcup_{i \in I} S_{i}$ is a $\wedge$-closed subset of $L$ and the lattices $L / S$ and colim ${ }_{i \in I} L / S_{i}$ are isomorphic (we shall identify these isomorphic lattices).

For any $a \in L$ we shall denote by $L_{a}$ the lattice of fractions $L /[a)$ associated with the filter [a) generated by $\{a\}$. If $x \in L$, then we shall denote by $x / a$ the congruence class $x /[a)$. Let $\pi_{a}: L \rightarrow L_{a}$ be the canonical morphism defined by $\pi_{a}(x)=x / a$, for all $x \in L$.

Remark 3.4. If $S$ is a $\wedge$-closed subset of $L$ then $(S, \geq)$ is a direct ordered set and the family $\left(L_{a}\right)_{a \in S}$ provides an inductive system of bounded distributive lattices. We observe that $[S)=\bigcup_{a \in S} S_{a}$, so, by applying Lemma 3.3 one gets $L / S=\operatorname{colim}_{a \in S} L_{a}$.

Let $P$ be a prime filter of $L$. Then $S=L-P$ is a filter. In this case we denote by $L_{P}=L /(L-P)$ the lattice of fractions of $L$ w.r.t. the $\wedge$-closed subset $L-P$. For any $a \in L,[a]_{P}$ will denote the congruence class of $a$ (modulo $\equiv_{L-P}$ ). Then $\pi_{P}: L \rightarrow L_{P}$ will be the canonical morphism defined by $\pi_{P}(a)=[a]_{P}$, for all $a \in L$.

Consider the continuous map $\pi_{P}^{*}: \operatorname{Spec}\left(L_{P}\right) \rightarrow \operatorname{Spec}(L)$ associated with $\pi_{P}$. If we denote $\Lambda(P)=\{Q \in \operatorname{Spec}(L) \mid Q \subseteq P\}$, then $\operatorname{Im}\left(\pi_{P}^{*}\right)=\Lambda(P)$. From Lemma 3.2 on gets:

Lemma 3.5. $\pi_{P}^{*}: \operatorname{Spec}\left(L_{P}\right) \rightarrow \operatorname{Spec}(L)$ is a homeomorphism.

We remark that $\pi_{P}^{*}$ is also an order-isomorphism. Following [14], for any prime ideal $P$ of $L$ we consider the ideal: $O(P)=\{x \in L \mid x \wedge y=0$, for some $y \in L-P\}$.

The annihilator of an element $x \in L$ is the ideal $x^{\perp}=\{y \in L \mid x \wedge y=0\}$. Therefore for any $P \in \operatorname{Spec}(L)$ we have $O(P)=\left\{x \in L \mid x^{\perp} \nsubseteq P\right\}$.

Lemma 3.6. For all $x \in L$ the following equivalence holds: $x \in O(P)$ if and only if $\pi_{P}(x)=[0]_{P}$. 
Proof. If $x \in L$, then $\pi_{P}(x)=[0]_{P}$ if and only if $x \wedge t=0$, for some $t \in L-P$ if and only if $x \in O(P)$.

Recall that a minimal prime ideal of $L$ is a minimal element of $\operatorname{Spec}(L)$. The set $\operatorname{Min}(L)$ of minimal prime ideals of $L$ is said to be the minimal prime spectrum of $L$. By using Zorn's axiom it follows that for any $P \in \operatorname{Spec}(L)$ there exists $Q \in \operatorname{Min}(L)$ such that $Q \subseteq P$.

By using Lemma 3.6 one obtains new proofs of some known results on minimal prime ideals in bounded distributive lattices:

Corollary 3.7. For any $P \in \operatorname{Spec}(L)$ we have $O(P)=\bigcap \Lambda(P)$. If $P$ is a minimal prime ideal of $L$, then $O(P)=P$. If $P$ and $O(P)$ are prime ideals of $L$, then $O(P)$ is minimal prime.

Proof. Assume that $Q \in \Lambda(P)$ and $x \in O(P)$. Thus $x \wedge y=0$ for some $y \notin P$, hence $y \notin Q$, so $x \in Q$ because $Q$ is prime. It follows that $x \in \bigcap \Lambda(P)$, so $O(P) \subseteq \bigcap \Lambda(P)$.

If $x \in \bigcap \Lambda(P)$, then $\pi_{P}(x) \in \pi_{P}^{*}(Q)$ for all $Q \in \operatorname{Spec}(L)$ such that $Q \subseteq P$. Since $\pi_{P}^{*}$ : $\operatorname{Spec}\left(L_{P}\right) \rightarrow \operatorname{Spec}(L)$ is an order-isomorphism the following hold:

$\pi_{P}(x) \in \bigcap\left\{\pi_{P}^{*}(Q) \mid Q \in \Lambda(P)\right\}=\pi_{P}^{*}\left(\bigcap \operatorname{Spec}\left(L_{P}\right)\right)=\left\{[0]_{P}\right\}$.

Thus $\pi_{P}(x)=[0]_{P}$ so $x \in O(P)$ (cf. Lemma 3.6). Therefore the converse inclusion $\bigcap \Lambda(P) \subseteq$ $O(P)$ is proven, and so $O(P)=\bigcap \Lambda(P)$. The other two properties are easy consequences of this equality.

The following known lemma follows immediatelly from Corollary 3.7.

Lemma 3.8. If $P$ is a prime ideal of $L$, then the following are equivalent

(1) $P$ is a minimal prime ideal of $L$;

(2) For all $x \in P$, there exists $y \in L-P$ such that $x \wedge y=0$.

Proposition 3.9. Let $f: L \rightarrow A$ be an injective morphism in $\mathbf{D}_{01}$ and $P$ a minimal prime ideal of $L$. Then there exists $Q \in \operatorname{Spec}(A)$ such that $f^{-1}(Q)=P$.

Proof. We observe that $S=f(L-P)$ is a $\wedge$-closed subset of A. Let us assume that $0 \in[f(L-P))$, so $0=f\left(a_{1}\right) \wedge \ldots \wedge f\left(a_{n}\right)$ for some elements $a_{1}, \ldots, a_{n} \in L-P$. Denoting $a=a_{1}, \cdots, a_{n}$ it results that $a \in L-P$ and $f(a)=0=f(0)$, hence $a=0$. This contradiction shows that $0 \notin[f(L-P))=S$, so $A / S$ is a non-trivial lattice. Thus there exists a prime ideal $Q^{\prime}$ of $A$. According to Lemma 3.1, if $Q=\pi_{S}^{*}\left(Q^{\prime}\right)$, then we have $Q \in \operatorname{Spec}(A)$ and $Q^{\prime}=\pi_{S}(Q)$. Let us consider the following commutative diagram in $\mathbf{D}_{01}$ :

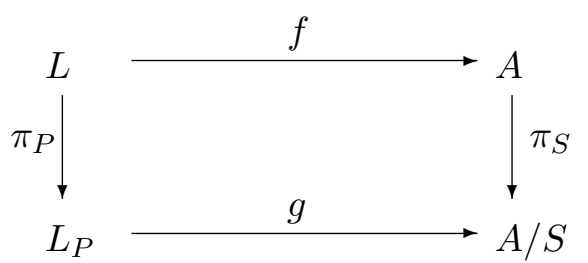

where $g: L_{P} \rightarrow A / S$ is the morphism defined by $g\left([x]_{P}\right)=f(x) / S$, for all $x \in L$. The above diagram induces the following commutative diagram in the category of topological spaces and continuous maps: 


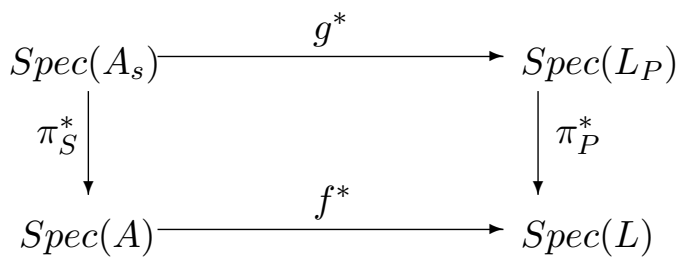

hence $f^{*}(Q)=f^{*}\left(\pi_{S}^{*}\left(Q^{\prime}\right)\right)=\pi_{P}^{*}\left(g^{*}\left(Q^{\prime}\right)\right) \subseteq P$, because $g^{*}\left(Q^{\prime}\right)$ is a prime ideal of $L$ and $\operatorname{Im}\left(\pi_{P}^{*}\right)=\Lambda(P)$. Since $P$ is a minimal prime ideal of $L$ and $f^{*}(Q) \in \operatorname{Spec}(L)$, we get $f^{*}(Q)=P$.

A bounded distributive lattice $L$ is said to be local if it has a unique maximal ideal (denoted by $\left.M_{L}\right)$.

Lemma 3.10. A bounded distributive lattice $L$ is local if and only if the following sentence holds in $L$ :

$$
\forall x y[x \wedge y=1 \Rightarrow x=1 \text { or } y=1] .
$$

Lemma 3.11. [10] If $P \in \operatorname{Spec}(L)$, then the lattice $L_{P}$ is local and its unique maximal ideal is $\pi_{P}(P)=\left\{[x]_{p} \mid x \in P\right\}$.

Lemma 3.12. If $L$ is a local lattice, then the quotient lattice $L / M_{L}$ is isomorphic to the Boolean algebra $L_{2}=\{0,1\}$.

Proof. By Lemma 1.1 of [10], $x \notin M_{L}$ implies $x=1$.

Corollary 3.13. If $P \in \operatorname{Spec}(L)$, then $L_{(P)}=L_{P} / \pi_{P}(P)$ is isomorphic to the Boolean algebra $L_{2}$.

Lemma 3.14. If $f: L \rightarrow A$ is morphism in $\mathbf{D}_{01}$, then the following properties are equivalent:

(1) $f\left(M_{L}\right) \subseteq M_{A}$;

(2) $f^{-1}\left(M_{A}\right)=M_{L}$;

(3) For all $x \in L, f(x)=1$ implies $x=1$.

If a morphism $f: L \rightarrow A$ fulfils the equivalent conditions (1)-(3) from Proposition 3.13, then it is called a local morphism.

Remark 3.15. For any $P \in \operatorname{Spec}(L)$ consider the map $\epsilon_{P}: L \rightarrow L_{(P)}=L_{2}$ defined by: For all $x \in L, \epsilon_{P}(x)=0$ if and only if $x \in P$. It is well-known that $\epsilon_{P}$ is a morphism in $\mathbf{D}_{01}$. Let us consider the following morphisms in $\mathbf{D}_{01}$ :

$$
L \stackrel{\pi_{P}}{\longrightarrow} L_{P} \stackrel{\nu_{P}}{\longrightarrow} L_{(P)}=L_{2},
$$

where $\nu_{P}\left([a]_{P}\right)=a / \pi_{P}(P)$, for all $a \in L$. It is easy to see that $\epsilon_{P}=\nu_{P} \circ \pi_{P}$.

Recall from [5] that the congruence $\sim_{I}$ associated with an ideal $I$ of $L$ has the following form: $x \sim_{I} y$ if and only if $x \vee t=y \vee t$ for some $t \in I$.

Lemma 3.16. Let $P$ be a prime ideal of $L$ and $x, y \in L$. If $x \sim_{O(P)} y$, then $x \equiv_{L-P} y$.

Proof. If $x \sim \sim_{O(P)} y$, then $x \vee t=y \vee t$ for some $t \in O(P)$. Thus there exists $s \in L-P$ such that $x \wedge t=0$, hence $x \wedge s=(x \vee t) \wedge s=(y \vee t) \wedge s=y \wedge s$. It follows that $x \equiv_{L-P} y$. 


\section{Flat morphisms of bounded distributive lattices}

In this section, we shall define the flat morphisms in the category $\mathbf{D}_{01}$ and we shall prove some of their basic properties. Firsly we shall present a description of flat ring morphisms that can be used as inspiration point in defining the flat lattice morphisms.

Let $R$ be a (unital) commutative ring and $I d(R)$ the quantale of ideals in $R$. Recall from [4], [8] the residuation operation in $I d(R)$ : For all ideals $I, J$ of $R,(I: J)=\{x \in R \mid x J \subseteq I\}$. In terms of [4],p.8, $(I: J)$ is the ideal quotient of $I$ and $J$. Similarly, if $I, J$ are ideals in a bounded distributive lattice $L$, then we define the residuation $(I: J)$ as the set of all $x \in L$ such that $x \wedge y \in I$, for all $y \in J$. If $J$ is the principal ideal (a] generated by a point set $\{a\}$, then we denote $(I: a)=(I:(a])$.

Let $\operatorname{Mod}_{R}$ be the category of $R$-modules. We fix an $R$-module $M$ and consider the functor $T_{M}: \operatorname{Mod}_{R} \rightarrow \operatorname{Mod}_{R}$ defined by $T_{M}(N)=N \bigotimes_{R} M$, for all $R$-module $N$. Following [4], p.29 we say that the $R$-module $M$ is flat if $T_{M}$ is an exact functor, i.e. transforms exact sequences into exact sequences. A ring morphisms $f: R \rightarrow S$ is flat if $S$ is flat as $R$-module.

If we want to define a notion of "flat morphisms" in an arbitrary category of algebras, then the previous definition of flat ring morphism is not very useful. Fortunately there exists a characterization of flat ring morphisms in terms of residuation operations of ideals.

According to Exercise 22 of [8], p.65 or [32], p.46, a ring morphism $f: R \rightarrow S$ is flat if and only if for each ideal $I$ of $R$ and for each finitely generated ideal $J$ of $R$ we have $(I: J) S=(I S: J S)$. By taking into account this equivalent definition of flat ring morphisms we are able to introduce a notion of flat morphism for the algebras whose lattices of congruences have a residuation operation. We shall apply this observation to define the flat morphisms of bounded distributive lattices.

Let $f: A \rightarrow B$ be a morphism of bounded distributive lattices. For each ideal $I$ of $A$ we denote by $f^{\bullet}(I)$ the ideal $(f(I)]$ of $B$ generated by $f(I)$. In this way we obtain a function $f^{\bullet}$ : $I d(A) \rightarrow I d(B)$ with the following property: For all $I \in I d(A)$ and $J \in I d(B), f^{\bullet}(I) \subseteq J$ if and only if $I \subseteq f^{*}(J)$. Thus $f^{\bullet}: \operatorname{Id}(A) \rightarrow \operatorname{Id}(B)$ is the left adjoint functor of $f^{*}: \operatorname{Id}(B) \rightarrow \operatorname{Id}(A)$ and $f^{\bullet}$ preseves the arbitrary joins. If $f: A \rightarrow B$ and $g: B \rightarrow C$ are two morphisms of $\mathbf{D}_{01}$, then $(g \circ f)^{\bullet}=g^{\bullet} \circ f^{\bullet}$.

We remind that any finitely generated ideal in a bounded distributive lattice is principal. Then a morphism $f: A \rightarrow B$ of $\mathbf{D}_{01}$ is said to be flat if for any ideal $I$ and $x \in A$ we have $f^{\bullet}(I: x)=$ $\left(f^{\bullet}(I): f(x)\right)$.

Lemma 4.1. If $f: A \rightarrow B$ is a morphism of $\mathbf{D}_{01}$, then the following are equivalent:

(1) $f$ is flat;

(2) For all ideals $I$ of $A$ and $x \in A$ we have $\left(f^{\bullet}(I): f(x)\right) \subseteq f^{\bullet}(I: x)$.

Proof. If $f: A \rightarrow B$ is an arbitrary morphism of $\mathbf{D}_{01}$, then it is easy to prove that for all ideals $I$ and $x \in A$ we have $f^{\bullet}(I: x) \subseteq\left(f^{\bullet}(I): f(x)\right)$.

Proposition 4.2. If $f: A \rightarrow B$ and $g: B \rightarrow C$ are two flat morphisms of $\mathbf{D}_{01}$, then $g \circ f$ is a flat morphism.

Proof. For all ideals $I$ of $A$ and $x \in A$ the following equalities hold:

$$
(g \circ f)^{\bullet}(I: x)=g^{\bullet}\left(f^{\bullet}(I: x)\right)=g^{\bullet}\left(f^{\bullet}(I): g(f(x))\right)=\left((g \circ f)^{\bullet}(I): g(f(x))\right) .
$$


Proposition 4.3. If $S$ is a $\wedge$-closed subset of the bounded distributive lattice $A$, then $\pi_{S}: A \rightarrow A / S$ is a flat morphism.

Proof. In accordance with Lemma 4.1 it suffices to prove that $\left(\pi_{S}^{\bullet}(I): x / S\right) \subseteq \pi_{S}^{\bullet}(I: x)$, for all ideals $I$ of $A$ and $x \in A$. By Lemma 3.1 we have $\pi_{S}^{\bullet}(I)=\{x / S \mid x \in I\}$ and $\pi_{S}^{\bullet}(I: x)=\{y / S \mid y \in$ $A, x \wedge y \in I\}$.

Let $y$ be an element of $A$ such that $y / S \in\left(\pi_{S}^{\bullet}(I): x / S\right)$, so $(x \wedge y) / S \in \pi_{S}^{\bullet}(I)$. Then there exists $z \in I$ such that $(x \wedge y) / S=z / S$, hence $x \wedge y \wedge t=z \wedge t$, for some $t \in S$. Since $z \wedge t \in I$, it follows that $y \wedge t \in(I: x)$. We remark that $y / S=y / S \wedge t / S=(y \wedge t) / S=\pi_{S}(y \wedge t)$. Thus $y / S \in \pi_{S}^{\bullet}(I: x)$, so $\left(\pi_{S}^{\bullet}(I): x / S\right) \subseteq \pi_{S}^{\bullet}(I: x)$. We conclude that $\pi_{S}$ is a flat morphism.

Lemma 4.4. If $f: A \rightarrow B$ is a morphism of $\mathbf{D}_{01}$, then the following are equivalent:

(1) $f$ is flat;

(2) For all $I \in \operatorname{Id}(A), x \in A$ and $y \in B$, the following implication holds:

$$
\exists a \in I[y \wedge f(x) \leq f(a)] \Rightarrow \exists b \in A[b \wedge x \in I, y \leq f(b)]
$$

Proof. For all $I \in I d(A), x \in A$ and $y \in B$, the following equivalences hold:

(i) $y \in\left(f^{\bullet}(I): f(x)\right)$ if and only if $y \wedge f(x) \in f^{\bullet}(I)$ if and only if $y \wedge f(x) \leq f(a)$, for some $a \in I$

and

(ii) $y \in f^{\bullet}(I: x)$ if and only if there exists $b \in(I: x)$ such that $y \leq f(b)$ if and only if there exists $b \in A$ such that $b \wedge x \in I$ and $y \leq f(b)$.

The previous equivalences together Lemma 4.1 imply that the properties (1) and (2) are equivalent.

The previous proposition offers a criterion to check if a morphism of $\mathbf{D}_{01}$ is flat.

Lemma 4.5. If $S$ is a $\wedge$-closed subset of a bounded distributive lattice $A$ and $f: A / S \rightarrow B$ is a morphism of $\mathbf{D}_{01}$, then the following properties are equivalent:

(1) $f$ is flat;

(2) $f \circ \pi_{S}$ is flat.

Proof. (1) $\Rightarrow(2)$ By Propositions 4.2 and 4.3 .

$(2) \Rightarrow(1)$ Let $J$ be an ideal of $A / S, z \in A / S$ and $y \in B$, so $I=\pi_{S}^{-1}(J) \in I d(A)$ and there exists $x \in A$ and there exists $x \in A$ such that $z=\pi_{S}(x)$. By Lemma 3.1(2) we have $J=\pi_{S}^{\bullet}(I)$. Assume that there exists $a \in A$ such that $\pi_{S}(a) \in J$ and $y \wedge f\left(\pi_{S}(a)\right) \leq f\left(\pi_{S}(x)\right)$. We observe that $a \in I$. Since $f \circ \pi_{S}$ is flat, there exists $b \in A$ such that $b \wedge x \in I$ and $y \leq f\left(\pi_{S}(b)\right.$ ) (cf. Proposition 4.4). Then $\pi_{S}(b) \wedge z=\pi_{S}(b \wedge x)$ is an element of $\pi_{S}^{\bullet}(I)=J$. By using again Proposition 4.4 it follows that $f$ is flat.

Lemma 4.6. Let $f: A \rightarrow B$ be a morphism of $\mathbf{D}_{01}, Q \in \operatorname{Spec}(B)$ and $P=f^{-1}(Q) \in \operatorname{Spec}(A)$. For all $x, y \in A$ the following implication holds:

$$
x \equiv_{A-P} y \Rightarrow f(x) \equiv_{B-Q} f(y) .
$$


Proof. Assume that $x \equiv_{A-P} y$, so $x \wedge t=y \wedge t$, for some $t \in A-P$. Then $f(t) \in B-Q$ and $f(x) \wedge f(t)=f(y) \wedge f(t)$, so $f(x) \equiv_{B-Q} f(y)$.

Let $f: A \rightarrow B$ be a morphism of $\mathbf{D}_{01}, Q \in \operatorname{Spec}(B)$ and $P=f^{-1}(Q) \in \operatorname{Spec}(A)$. According to Lemma 4.6, one can define a map $f_{Q}: A_{P} \rightarrow B_{Q}$ by $f_{Q}\left([x]_{P}\right)=[f(x)]_{Q}$, for each $x \in a$. Then $f_{Q}$ is a morphism of bounded distributive lattices and the following diagram is commutative:

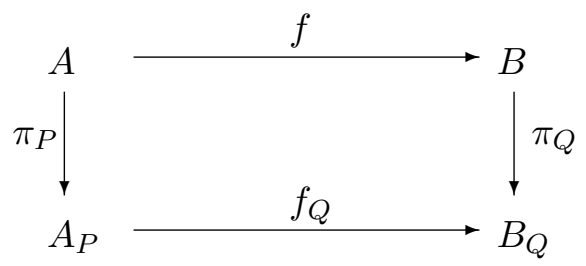

Proposition 4.7. If $f: A \rightarrow B$ is flat, then $f_{Q}: A_{P} \rightarrow B_{Q}$ is a flat morphism.

Proof. Assume that $f: A \rightarrow B$ is flat. By Proposition 4.3, $\pi_{P}$ and $\pi_{Q}$ are flat morphisms. According to Proposition 4.2, $\pi_{q} \circ f$ is flat. By using the previous commutative diagram it follows that $f_{Q} \circ \pi_{P}$ is flat, therefore, in accordance with Lemma 4.5, we conclude that $f_{Q}$ is a flat morphism.

Proposition 4.8. Let $A, B$ be two non-trivial bounded distributive lattices and $f: A \rightarrow B$ be a morphism in $\mathbf{D}_{01}$. The following are equivalent:

(1) $f$ is flat;

(2) For any $Q \in \operatorname{Spec}(B)$, the morphism $f_{Q}: A_{f^{-1}(Q)} \rightarrow B_{Q}$ is flat;

(3) For any $N \in \operatorname{Max}(B)$, the morphism $f_{N}: A_{f^{-1}(N)} \rightarrow B_{N}$ is flat.

Proof. (1) $\Rightarrow(2)$ By Proposition 4.7.

$(2) \Rightarrow(3)$ Obviously.

$(3) \Rightarrow(1)$ Since $B$ is a non-trivial bounded distributive lattice there exists a maximal ideal $N$ in $B$. Let us consider the following commutative diagram

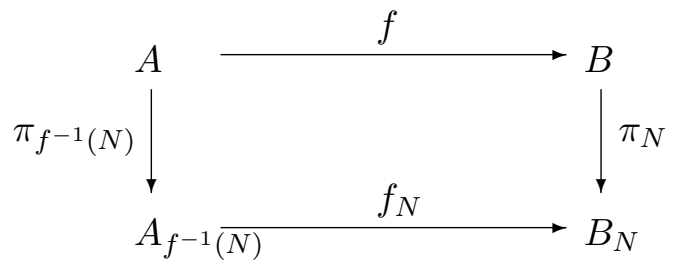

The morphisms $\pi_{f^{-1}(N)}, \pi_{N}$ and $f_{N}$ are flat, therefore by using Propositions 4.2 and 4.5, from $\pi_{N} \circ f=f_{N} \circ \pi_{f^{-1}(N)}$ it follows that $f$ is flat.

Assume that $\left(L_{i}, u_{i j}: L_{i} \rightarrow L_{j}\right)_{i \leq j}$ is an inductive system in the category of bounded distributive lattices, $\operatorname{colim}_{i \in I} L_{i}$ is its colimit and $u_{i}: L_{i} \rightarrow \operatorname{colim}_{i \in I} L_{i}, i \in I$ are the canonical morphisms. Let $f_{i}: L_{i} \rightarrow A, i \in I$ be a family of morphisms such that $f_{j} \circ u_{i j}=f_{i}$, for all $i \leq j$ and $f: \operatorname{colim}_{i \in I} L_{i} \rightarrow A$ the induced morphism. Recall that $f \circ u_{i}=f_{i}$, for all $i \in I$. 
Proposition 4.9. If the morphisms $f: L_{i} \rightarrow A, i \in I$ are flat, then the morphism $f:$ colim $_{i \in I} L_{i} \rightarrow$ $A$ is flat.

Proof. Let $J$ be an ideal of $\operatorname{colim}_{i \in I} L_{i}, x \in \operatorname{colim}_{i \in I} L_{i}$ and $y \in A$. Suppose that there exists $a \in J$ such that $y \wedge f(x) \leq f(a)$. According to the construction of the colimit there exist $i \in I$ and $c, d \in L_{i}$ such that $x=u_{i}(c)$ and $a=u_{i}(d)$. We remark that $u_{i}^{-1}(J)$ is an ideal of the lattice $L_{i}$, $d \in u_{i}^{-1}(J), f(x)=f\left(u_{i}(c)\right)=f_{i}(c)$ and $f(a)=f\left(u_{i}(d)\right)=f_{i}(d)$, hence $y \wedge f_{i}(c) \leq f_{i}(d)$. Since the morphism $f_{i}$ is flat, by Proposition 4.4, there exists an element $e \in L_{i}$ such that $e \wedge c \in u_{i}^{-1}(J)$ and $y \leq f_{i}(e)$. Denoting $b=u_{i}(e)$ we have $b \in \operatorname{colim}_{i \in I} L_{i}$ and the following implications hold:

(a) $e \wedge c \in u_{i}^{-1}(J) \Rightarrow b \wedge x=u_{i}(e) \wedge u_{i}(c)=u_{i}(e \wedge c) \in J$

(b) $f_{i}(e)=f\left(u_{i}(e)\right)=f(b) \Rightarrow y \leq f_{i}(e)=f(b)$.

In accordance with Proposition 4.4, from (a) and (b) it follows that $f$ is a flat morphism.

Let $J$ be an ideal of a bounded distributive lattice $L$ and $p_{J}: L \rightarrow L / J$ the canonical surjective morphism. Then $[J)=\{I \in I d(L) \mid J \subseteq I\}$ is a frame and the function $\Phi:[J) \rightarrow I d(L / J)$ is defined by $I \mapsto I / J$, is a frame isomorphism. If $I, K \in[J)$, then the residuation $(I: K)_{J}$ in the frame $[J)$ has the form $(I: K)_{J}=(I: K) \vee J$. Let us consider the function $\Psi: I d(L) \rightarrow[J)$, defined by $\Psi(I)=I \vee J$, for all $I \in I d(L)$. Then the following diagram is commutative:

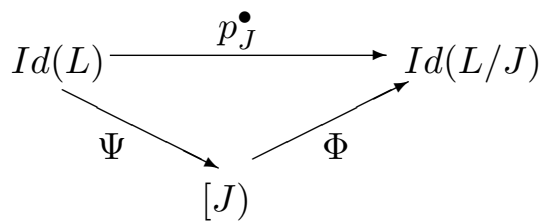

Lemma 4.10. For any ideal $J$ of $L$ the following properties are equivalent:

(1) $p_{J}: L \rightarrow L / J$ is flat;

(2) For all $I \in I d(L)$ and $x \in L,((I \vee J): x) \subseteq(I: x) \vee J$.

Proof. If $I \in I d(L)$ and $x \in L$, then by using the properties of residuation we $\operatorname{get}((I \vee J)$ : $((x] \vee J))=((I \vee J): x)$. Then by using Lemma 4.1 and the above commutative diagram (where $\Phi$ is a frame isomorphism) the following assertions are equivalent:

(a) $p_{J}$ is flat:

(b) For all $I \in I d(L)$ and $x \in L,\left(p_{J}^{\bullet}(I): p_{J}(x)\right) \subseteq p_{J}^{\bullet}(I: x)$

(c) For all $I \in I d(L)$ and $x \in L,\left(\Psi(I): \Psi((x])_{J} \subseteq \Psi(I: x)\right.$

(d) For all $I \in I d(L)$ and $x \in L,((I \vee J):((x] \vee J))_{J} \subseteq(I: x) \vee J$

(e) For all $I \in I d(L)$ and $x \in L,((I \vee J): x) \vee J \subseteq(I: x) \vee J$

(f) For all $I \in I d(L)$ and $x \in L,((I \vee J): x) \subseteq(I: x) \vee J$.

Recall from [14], [22] that an ideal $J$ of a bounded distributive lattice $L$ is a $\sigma$-ideal if $J \vee x^{\perp}=L$, for $x \in J$.

Proposition 4.11. For each ideal $J$ of $L$ the following properties are equivalent:

(1) $p_{J}: L \rightarrow L / J$ is a flat morphism;

(2) $J$ is a $\sigma$-ideal. 
Proof. (1) $\Rightarrow(2)$ Assume that $p_{J}: L \rightarrow L / J$ is a flat morphism. By taking $I=\{0\}$ in Lemma $4.10,(2)$ we obtain $(J: x) \subseteq J \vee x^{\perp}$. If $x \in J$, then $(J: x)=L$, hence $J \vee x^{\perp}=L$. Thus $J$ is a $\sigma$-ideal.

$(2) \Rightarrow(1)$ Let $J$ be a $\sigma$-ideal and $a \in((I \vee J): x)$, hence $a \wedge x \in I \vee J$. Then there exist $c \in I$ and $d \in J$ such that $a \wedge x=c \vee d$. Since $J$ is a $\sigma$-ideal we have $J \vee d^{\perp}=L$, hence there exist $e \in J$ and $f \in d^{\perp}$ such that $e \vee f=1$. Thus $d \wedge f=0$, so

$$
a \wedge f \wedge x=(c \vee d) \wedge f=(c \wedge f) \vee(d \wedge f)=c \wedge f .
$$

We observe that $c \wedge f \in I$, hence $a \wedge f \in(I: x)$. From $a=a \wedge(e \vee f)=(a \wedge e) \vee(a \wedge f), a \wedge e \in J$ and $a \wedge f \in(I: x)$ we get that $a \in(I: x) \vee J$. We have proven that $((J \vee J): x) \subseteq(I: x) \vee J$, therefore, by using Lemma 4.10, it follows that $p_{J}$ is flat.

According to Lemma 3.6, one can define a function $u_{P}: L / O(P) \rightarrow L_{P}$ by $u_{P}(x / O(P))=[x]_{P}$, for all $x \in L$. Then $u_{P}$ is a surjective morphism of $\mathbf{D}_{01}$ and the following diagram is commutative:

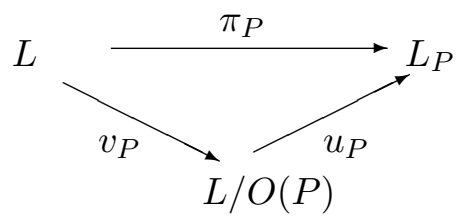

where $v_{P}: L \rightarrow L / O(P)$ is the lattice morphism defined by $u_{P}(x)=x / O(P)$, for all $x \in L$.

In the following proposition we shall keep the notations from the previous commutative diagram.

Proposition 4.12. If $v_{P}$ is a flat morphism, then $u_{P}$ is flat.

Proof. Let $J$ be an ideal of $L / O(P)$ and $x \in L$. Then there exists an ideal $I$ of $L$ such that $v_{P}^{\bullet}(I)=J$. By Proposition 4.3, $\pi_{P}$ is a flat morphism, hence, by taking into account that $v_{P}$ is flat, the following equalities hold:

$$
\begin{aligned}
\left(u_{P}^{\bullet}(J): u_{P}(x / P)\right) & =\left(u_{P}^{\bullet}\left(v_{P}^{\bullet}(I)\right): u_{P}\left(v_{P}(x)\right)\right)=\left(\pi_{P}^{\bullet}(I): \pi_{P}(x)\right)=\pi_{P}^{\bullet}(I: x) \\
& =u_{P}^{\bullet}\left(v_{P}^{\bullet}(I: x)\right)=u_{P}^{\bullet}\left(v_{P}^{\bullet}(I): v_{P}(x)\right)=u_{P}^{\bullet}(J: x / O(P)) .
\end{aligned}
$$

We conclude that $u_{P}$ is a flat morphism.

Corollary 4.13. Let $P$ be a prime ideal of $L$ such that $O(P)$ be a $\sigma$-ideal. Then $u_{P}$ is a flat morphism.

Proof. By Propositions 4.11 and 4.12 .

\section{Patch and flat topologies on the prime spectrum}

Let us fix a bounded distributive lattice $L$. If we endow the prime spectrum $\operatorname{Spec}(L)$ with the Stone topology then we obtain a spectral topological space, denoted by $\operatorname{Spec}_{(} L$ ) (see [5], [27]). Following [24], [16], [27] one can define on $\operatorname{Spec}(L)$ two important topologies:

- the patch topology, having as basis the family $(D(a) \cap V(b))_{a, b \in L}$; 
- the flat topology, having as basis the family $(V(b))_{b \in L}$.

We shall denote by $\operatorname{Spec}_{P}(L)\left(\operatorname{resp} . \operatorname{Spec}_{F}(L)\right)$ the prime spectrum $\operatorname{Spec}(L)$ endowed with the patch topology (resp. the flat topology). According to [16] and [27], $\operatorname{Spec}_{P}(L)$ is a Boolean space and $\operatorname{Spec}_{F}(L)$ is a spectral space. If $L$ is a Boolean algebra, then Stone topology, flat topology and patch topology are identical.

Lemma 5.1. [16] If $f: A \rightarrow B$ is a morphism of bounded distributive lattices, then $f^{*}: \operatorname{Spec}(B) \rightarrow$ $\operatorname{Spec}(A)$ is both patch and flat continuous.

Now, we shall describe the closed subsets of $\operatorname{Spec}_{F}(L)$ in terms of lattices of fractions.

Proposition 5.2. If $S$ is a $\wedge$-closed subset of $L$, then $\operatorname{Im}\left(\pi_{S}^{*}\right)$ is closed subset of $\operatorname{Spec}_{F}(L)$.

Proof. We shall prove that $U=\operatorname{Spec}(L)-\operatorname{Im}\left(\pi_{S}^{*}\right)$ is an open subset of $\operatorname{Spec}_{F}(L)$. Recall from Section 3 that $\operatorname{Im}\left(\pi_{s}^{*}\right)=\{P \in \operatorname{Spec}(A) \mid P \cap S=\emptyset\}$, so for each $P \in \operatorname{Spec}(L)$, the following equivalences hold: $P \in U$ if and only if $P \bigcap S \neq \emptyset$ if and only if there exists an element $s \in P \cap S$. Let us consider a point $P \in U$, hence there exists an element $s$ of $P \bigcap S$. If $Q \in V(s)$, then $s \in Q \bigcap S$, hence $Q \in U$. Thus $P \in V(s) \subseteq U$ and $V(s)$ is a basic open subset of $\operatorname{Spec}_{F}(L)$, hence $U$ is an open subset of $\operatorname{Spec}_{F}(L)$.

Proposition 5.3. If $E$ is a closed subset of $\operatorname{Spec}_{F}(L)$, then there exists a $\wedge$-closed subset of $L$ such that $E=\operatorname{Im}\left(\pi_{S}^{*}\right)$.

Proof. (a) Firstly we consider the particular case of a basic open subset $E=D(a)$, where $a$ is an element of $L$. Let us take the canonical morphism $\pi_{a}: L \rightarrow L_{a}$. Recall that $L_{a}$ is the lattice of fractions of $L$ associated with the principal filter $[a)$. From Section 3 we know that $\operatorname{Im}\left(\pi_{a}^{*}\right)$ $=\{P \in \operatorname{Spec}(A) \mid P \bigcap[a)=\emptyset\}$, therefore for any $Q \in \operatorname{Spec}(L)$ the following equivalences hold: $Q \in \operatorname{Im}\left(\pi_{a}^{*}\right)$ if and only if $Q \bigcap[a)=\emptyset$ if and only if $a \notin Q$ if and only if $Q \in D(a)$. Hence $\operatorname{Im}\left(\pi_{S}^{*}\right)$ is exactly the flat closed set $D(a)$.

(b) In general, a closed subset $E$ of $\operatorname{Spec}_{F}(L)$ has the form $E=\bigcap_{i \in I} D\left(a_{i}\right)$, for some family $\left(a_{i}\right)_{i \in I} \subseteq L$. Let $S_{\omega}(I)$ be the family of finite subsets of $I$. For each $J \in S_{\omega}(I)$ let us denote $b_{J}=\bigwedge_{i \in J} a_{i}$. It is easy to see that $S=\left\{b_{j} \mid J \in S_{\omega}(I)\right.$ is a $\wedge$ - closed subset of $L$. According to (a), for each $J \in S_{\omega}(I)$ we have $D\left(b_{J}\right)=\operatorname{Im}\left(\pi_{b_{J}}^{*}\right)$. By taking into account Remark 3.4, $\operatorname{Spec}(L / S)=\operatorname{Spec}\left(\operatorname{colim}_{J \in S_{\omega}(I)} L_{b_{J}}\right)$, so by using Corollary 2.3, the following equalities hold:

$$
\begin{aligned}
\operatorname{Im}\left(\pi_{S}^{*}\right) & =\pi_{S}^{*}(\operatorname{Spec}(L / S))=\pi_{S}^{*}\left(\operatorname{Spec}\left(\operatorname{colim}_{J \in S_{\omega}(I)} L_{b_{J}}\right)\right)=\bigcap\left\{\operatorname{Im}\left(\pi_{b_{J}}^{*}\right) \mid J \in S_{\omega}(I)\right\} \\
& =\bigcap\left\{D\left(b_{J}\right) \mid J \in S_{\omega}(I)\right\}=\bigcap_{i \in I} D\left(a_{i}\right)=E .
\end{aligned}
$$

Theorem 5.4. For any subset $E$ of $\operatorname{Spec}(L)$ the following properties are equivalent:

(1) $E$ is a closed subset of $\operatorname{Spec}_{F}(L)$;

(2) There exists a $\wedge$-closed subset $S$ of $L$ such that $E=\operatorname{Im}\left(\pi_{S}^{*}\right)$.

Proof. By Propositions 5.2 and 5.3.

Corollary 5.5. If $E$ is a closed subset of $\operatorname{Spec}_{F}(L)$, then there exists a flat morphism $f: L \rightarrow A$ such that $E=\operatorname{Im}\left(f^{*}\right)$. 
Proof. By Propositions 5.3 and 4.3.

We don't know if the converse of Corollary 5.5 holds: $\operatorname{Im}\left(f^{*}\right)$ is a flat subset of $\operatorname{Spec}(L)$ for any flat lattice morphism $f: L \rightarrow A$ ?

The previous results can be used in obtaining new proofs for most of properties of flat topology.

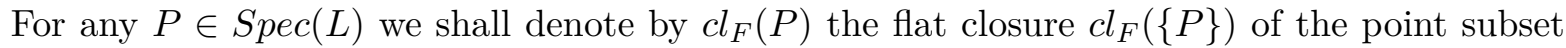
$\{P\}$ of $\operatorname{Spec}(L)$.

Proposition 5.6. For any prime ideal $P$ of $L$ we have $\operatorname{cl}_{F}(P)=\Lambda(P)$.

Proof. By applying Proposition 5.2, $\Lambda(P)=\operatorname{Im}\left(\pi_{P}^{*}\right)$ is a flat closed subset of $\operatorname{Spec}(L)$. Assume that $E$ is a closed subset of $\operatorname{Spec}_{F}(L)$ such that $P \in E$. According to Proposition 5.3 there exists a $\wedge$-closed subset of $L$ such that $E=\operatorname{Im}\left(\pi_{S}^{*}\right)=\{Q \in \operatorname{Spec}(L) \mid Q \bigcap S=\emptyset\}$. From any $P \in E$ we get $P \cap S=\emptyset$, hence the following implications hold:

$$
Q \in \Lambda(P) \Rightarrow Q \subseteq P \Rightarrow Q \bigcap S=\emptyset \Rightarrow Q \subseteq E .
$$

This shows that $\Lambda(P) \subseteq E$, hence $c l_{F}(P)=\Lambda(P)$.

Corollary 5.7. If $E$ is a compact subset of $\operatorname{Spec}_{Z}(L)$, then the flat closure $l_{F}(E)$ of $E$ is $c l_{F}(E)=\bigcup_{P \in E} \Lambda(P)$.

Proof. The corollary follows from Proposition 5.6 by using a similar argument as in the proof of Lemma 3.7 of [1].

Now, we shall establish a characterization theorem for the closed subsets of $\operatorname{Spec}_{P}(L)$.

Lemma 5.8. If $f: L \rightarrow A$ is an arbitrary morphism of bounded distributive lattices, then $\operatorname{Im}\left(f^{*}\right)$ is a closed subset of $\operatorname{Spec}_{P}(L)$.

Proof. By Lemma 5.1, $f^{*}: \operatorname{Spec}(A) \rightarrow \operatorname{Spec}(L)$ is patch continuous. The topological space $\operatorname{Spec}_{P}(A)$ is compact, hence its image $\operatorname{Im}\left(f^{*}\right)$ is a compact subset of the Boolean space $\operatorname{Spec}_{P}(L)$. Then $\operatorname{Im}\left(f^{*}\right)$ is a closed subset of $\operatorname{Spec}_{P}(L)$.

Let $E$ be a subset of $\operatorname{Spec}(L)$ and $\epsilon: L \rightarrow \prod_{P \in E} L_{P} / \pi_{P}(P)=L_{2}^{E}$ the lattice morphism is defined by $\epsilon(P)=\left(\epsilon_{P}(x)\right)_{P \in E}$ (see the notations from Remark 3.13). Thus $\epsilon^{*}: \operatorname{Spec}\left(L_{2}^{E}\right) \rightarrow$ $\operatorname{Spec}(L)$ is patch continuous.

The following result is the lattice version of Theorem 3.1 of [35].

Proposition 5.9. The patch closure of $E \subseteq \operatorname{Spec}(L)$ is $\operatorname{cl}_{P}(E)=\operatorname{Im}\left(\epsilon^{*}\right)$.

Proof. For each $P \in E$ let us consider the projection $p r_{P}: L_{2}^{E} \rightarrow L_{2}$, is defined by $\operatorname{pr}_{P}(a)=a_{P}$, for each element $a=\left(a_{P}\right)_{P \in E}$ of $L_{2}^{E}$. Thus $I_{P}=p r_{P}^{-1}(0)=\left\{a \in L_{2}^{E} \mid a_{P}=0\right\}$ is a prime ideal of $L_{2}^{E}$. For each $x \in L$ the following equivalences hold:

$$
x \in \epsilon^{-1}\left(I_{P}\right) \text { iff } \epsilon(x) \in I_{P} \text { iff }\left(\epsilon_{Q}(x)\right)_{Q \in E} \in I_{P} \text { iff } \epsilon_{P}(x)=0 \text { iff } x \in P,
$$

hence $P=\epsilon^{-1}\left(I_{P}\right)=\epsilon^{*}\left(I_{P}\right)$, for all $P \in E$. Therefore we get the inclusion $E \subseteq \operatorname{Im}\left(\epsilon^{*}\right)$. In accordance with Lemma 5.8, $\operatorname{Im}\left(\epsilon^{*}\right)$ is patch closed, so $\operatorname{cl}_{P}(E) \subseteq \operatorname{Im}\left(\epsilon^{*}\right)$.

Now, we shall establish the converse inclusion $\operatorname{Im}\left(\epsilon^{*}\right) \subseteq c l_{P}(E)$. Firstly we shall prove that $E^{\prime}=\left\{I_{P} \mid P \in E\right\}$ is a dense subset of $\operatorname{Spec}_{P}\left(L_{2}^{E}\right)$. Since $L_{2}^{E}$ is a Boolean algebra, the patch 
topology of $\operatorname{Spec}\left(L_{2}^{E}\right)$ coincides with the Stone topology, so it suffices to show that any non-empty basic open set U in Stone's topology of $\operatorname{Spec}\left(L_{2}^{E}\right)$ intersects $E^{\prime}$. One can assume that $U=D(x)$, where $x$ is a non-zero element of $L_{2}^{E}$. Then there exists $Q \in E$ such that $x \notin I_{Q}$, i.e. $I_{Q} \in D(x)$. Since $I_{Q} \in E^{\prime}$ it follows that $I_{Q} \in E^{\prime} \cap D(x)$, therefore $\operatorname{cl}_{P}\left(E^{\prime}\right)=\operatorname{Spec}_{P}\left(L_{2}^{E}\right)$.

We remark that

$$
\epsilon^{*}\left(E^{\prime}\right)=\left\{\epsilon^{-1}\left(I_{Q}\right) \mid Q \in E\right\}=\{Q \mid Q \in E\}=E,
$$

hence $\epsilon^{*}\left(E^{\prime}\right) \subseteq c l_{P}(E)$. It follows that $E^{\prime} \subseteq\left(\epsilon^{*}\right)^{-1}\left(c l_{P}(E)\right)$. Since $\left(\epsilon^{*}\right)^{-1}\left(c_{P}(E)\right)$ is closed in $\operatorname{Spec}_{P}\left(L_{2}^{E}\right)$, we get $\left.c l_{P}\left(E^{\prime}\right) \subseteq \epsilon^{*}\right)^{-1}\left(c_{P}(E)\right)$, hence $\epsilon^{*}\left(c_{P}\left(E^{\prime}\right)\right) \subseteq c l_{P}(E)$. Thus we obtain

$$
\operatorname{Im}\left(\epsilon^{*}\right)=\epsilon^{*}\left(\operatorname{Spec}_{P}\left(L_{2}^{E}\right)\right)=\epsilon^{*}\left(\operatorname{cl}_{P}\left(E^{\prime}\right)\right) \subseteq \operatorname{cl}_{P}(E) .
$$

Theorem 5.10. For any subset $E$ of $\operatorname{Spec}(L)$ the following properties are equivalent:

(1) E is a closed subset of $\operatorname{Spec}_{P}(L)$;

(2) There exists a morphism $f: L \rightarrow A$ of $\mathbf{D}_{01}$ such that $E=\operatorname{Im}\left(f^{*}\right)$.

Proof. By Lemma 5.8 and Proposition 5.9.

\section{Applications to conormal and Stone lattices}

The conormal lattices were introduced in [13] under the name of normal lattices (for this terminology see the discussion of [27], p.78). Recall that a bounded distributive lattice $L$ is said to be conormal if for all $a, b \in L$ such that $a \wedge b=0$ there exist $x, y \in L$ such that $a \wedge x=b \wedge y=0$ and $x \vee y=1$.

The following proposition collects several properties that characterize the conormal lattices.

Proposition 6.1. [13] If $L$ is a bounded distributive lattice, then the following are equivalent:

(1) L is a conormal lattice;

(2) If $P$ and $Q$ are distinct minimal prime ideals of $L$, then $P \vee Q=L$;

(3) Each prime ideal of $L$ contains a unique minimal prime ideal;

(4) Any minimal prime ideal of $L$ is a $\sigma$-ideal;

(5) If $x, y \in L$, then $x \wedge y=0$ implies $x^{\perp} \vee y^{\perp}=L$;

(6) For all $x, y \in L$ we have $(x \wedge y)^{\perp}=x^{\perp} \vee y^{\perp}$;

(7) For each $x \in L, x^{\perp}$ is a $\sigma$-ideal.

Let us fix a bounded distributive lattice $L$. Then $L$ is called dense if for all $x, y \in L, x \wedge y=0$ implies $x=0$ or $y=0$. It is easy to see that $L$ is dense if and only if $\{0\}$ is a prime ideal if and only if $L$ has a unique minimal prime ideal.

Theorem 6.2. If $L$ is a bounded distributive lattice, then the following properties are equivalent:

(1) L is a conormal lattice; 
(2) For all distinct minimal prime ideals $P$ and $Q$ of $L, O(P) \vee O(Q)=L$;

(3) For each prime ideal $P$ of $L, L_{P}$ is a dense lattice;

(4) For each maximal ideal $M$ of $L, L_{M}$ is a dense lattice;

(5) For each $P \in \operatorname{Spec}(L), O(P)$ is a prime ideal;

(6) For each $M \in \operatorname{Max}(A), O(M)$ is a prime ideal;

(7) For all $P, Q \in \operatorname{Spec}(L), P \subseteq Q$ implies $O(P)=O(Q)$.

Proof. (1) $\Rightarrow(2)$ Let $P$ and $Q$ be two distinct minimal prime ideals of $L$. By Corollary 3.7, $P=O(P)$ and $Q=O(Q)$, hence, by using Proposition 6.1(2), we get $O(P) \vee O(Q)=L$.

$(2) \Rightarrow(1)$ If $P, Q \in \operatorname{Min}(A)$ and $P \neq Q$, then $P \vee Q=O(P) \vee O(Q)=L$.

$(1) \Rightarrow(5)$ Let $P$ be a prime ideal of $L$. In order to show that $O(P)$ is a prime ideal, assume that $x$ and $y$ are two elements of $L$ such that $x \wedge y \in O(P)$, hence $(x \wedge y)^{\perp} \not \subset P$. By Proposition 6.1(6) we have $(x \wedge y)^{\perp}=x^{\perp} \vee y^{\perp}$, so $x^{\perp} \vee y^{\perp} \nsubseteq P$. Thus $x^{\perp} \nsubseteq P$ and $y^{\perp} \nsubseteq P$, hence $x \in P$ or $y \in P$, because $P$ is prime. Conclude that $O(P)$ is prime.

$(5) \Rightarrow(3)$ Let $P$ be a prime ideal of $L$, so $O(P)$ is prime. According to Corollary 3.7 and Proposition 6.1(3), $O(P)$ is the unique minimal prime ideal of $L$ included in $P$. Since $\pi_{P}^{*}$ : $\operatorname{Spec}\left(L_{P}\right) \rightarrow \Lambda(P)$ is an order-isomorphism it follows that $\pi_{P}(O(P))$ is the unique minimal prime ideal of $L_{P}$, therefore $L_{P}$ is a dense lattice.

$(3) \Rightarrow(4)$ Obviously.

$(4) \Rightarrow(1)$ Assume by absurdum that there exist two distinct minimal prime ideals $P$ and $Q$ such that $P \vee Q \neq L$, so $P, Q \subseteq M$, for some maximal ideal $M$ of $L$. We know that $\operatorname{Spec}\left(L_{M}\right)$, $\Lambda(M)$ are order-isomorphic and $L_{M}$ is a dense lattice, so $\Lambda(M)$ has a unique minimal element. We have obtained a contradiction, hence $L$ is conormal (cf. Proposition 6.1(2)).

$(5) \Rightarrow(6)$ Obviously.

$(6) \Rightarrow(1)$ Let $P$ be a prime ideal of $L$ and $M \in \operatorname{Max}(A)$ such that $P \subseteq M$. Consider an arbitrary minimal prime ideal $Q$ such that $Q \subseteq P$. Thus $Q=O(Q) \subseteq O(M)$ and $O(M)=\bigcap \Lambda(M)$ is prime, so $Q$ and $O(M)$ are two minimal prime ideals. It follows that $Q=O(M)$, so $P$ contains a unique minimal prime ideal.

$(5) \Rightarrow(7)$ Assume that $P, Q \in \operatorname{Spec}(L)$ and $P \subseteq Q$, hence $O(P) \subseteq O(Q)$. By Corollary 3.7, $O(P)$ and $O(Q)$ are minimal prime, so $O(P)=O(Q)$.

$(7) \Rightarrow(1)$ Let $P$ and $Q$ two distinct minimal prime ideals of $L$. Assume that $P \vee Q \neq L$, so $P \vee Q \subseteq M$, for some maximal ideal $M$. Applying the hypothesis (7) and Corollary 3.7 one obtains $P=O(P)=O(M)=O(Q)=Q$, contradicting that $P, Q$ are distinct. Therefore $P \vee Q=L$, hence $L$ is conormal (cf. Proposition 6.1(2)).

Remark 6.3. By [3], the reticulation of a commutative ring $R$ is a bounded distributive lattice $L(A)$ whose prime spectrum Spec $(L(R))$ is homeomorphic to the prime spectrum Spec $(R)$ of $R$. In fact, we have a reticulation functor from the category Rings of commutative rings to the category $\mathbf{D}_{01}$. The reticulation functor allows us to transport some results from $\mathbf{D}_{01}$ to Rings and viceversa. Then the previous result can be viewed as a lattice version of Theorem 3.2 of [39] and each of these two results can be obtained as a consequence of the other (by using the reticulation functor).

Corollary 6.4. Let $L$ be a conormal lattice and $P \in \operatorname{Spec}(L)$. Then $O(P)$ is the unique prime ideal of $L$ included in $P$. 
Proof. By Theorem 6.2(5), $O(P)$ is a prime ideal, therefore, by applying Proposition 6.1(3), it follows that $O(P)$ is the unique minimal prime ideal of $L$ included in $P$.

Let us consider the topological space $\operatorname{Min}_{F}(L)$ obtained by restricting the flat topology of $\operatorname{Spec}(L)$ to the minimal prime spectrum $\operatorname{Min}(L)$. If we apply Theorem 8.14 of [21] to the frame $\operatorname{Id}(L)$ of ideals in $L$, then we get the following equivalence: $L$ is a conormal lattice if and only if the inclusion $\operatorname{Min}(L) \subseteq \operatorname{Spec}(L)$ has a flat continuous retraction $\operatorname{Spec}(L) \rightarrow \operatorname{Min}(L)$.

Corollary 6.5. Let $L$ be an arbitrary bounded distributive lattice. If $\gamma: \operatorname{Spec}(L) \rightarrow \operatorname{Min}(L)$ is a continuous flat retraction of the inclusion $\operatorname{Min}(L) \subseteq \operatorname{Spec}(L)$, then $\gamma(P)=O(P)$, for all $P \in \operatorname{Spec}(L)$.

Proof. Let $P$ be a prime ideal of $L$ and $Q$ a minimal prime ideal such that $Q \subseteq P$. By Proposition 5.6 we have $Q \in \Lambda(P)=c l_{F}(P)$. Since $\gamma$ is a continuous flat retraction of $\operatorname{Min}(L) \subseteq \operatorname{Spec}(L)$, it follows that

$$
Q=\gamma(Q) \in c_{F}(\gamma(P)) \bigcap \operatorname{Min}(L)=\Lambda(\gamma(P)) \bigcap \operatorname{Min}(L)=\{\gamma(P)\} .
$$

Thus $\gamma(P)=Q$, so $\gamma(P)$ is the unique minimal prime ideal of $L$ included in $P$. In accordance with Proposition 6.1(3), $L$ is a conormal lattice. Applying Corollary 6.4, we get $\gamma(P)=O(P)$.

Corollary 6.6. If $P$ is a minimal prime ideal of a conormal lattice $L$, then the morphism $u_{P}$ : $L / O(P) \rightarrow L_{P}$ is flat.

Proof. By Theorem 6.2(5), $O(P)$ is a prime ideal. According to Proposition 6.3(4), $O(P)$ is a minimal prime ideal, hence, by Corollary 4.13 , it follows that $u_{P}$ is a flat morphism.

Recall that a bounded distributive lattice $L$ is a Stone lattice if for any $x \in L$ there exists $e \in B(L)$ such that the annihilator ideal $x^{\perp}$ is equal to the principal ideal $(x]$ generated by $\{x\}$. In what follows we shall find new properties that characterize the Stone lattices.

We observe that $D=L-\left\{x \in L \mid x^{\perp}=\{0\}\right\}$ is a $\sigma$-closed subset of $L$. One can prove that $D=\bigcup \operatorname{Min}(L)$. Following [21] we shall denote by $T(L)$ the lattice of fractions $L / D$ and $\pi: L \rightarrow T(L)$ the canonical morphism $\pi(x)=x / D$, for all $x \in L$.

Proposition 6.7. [21] The following properties are equivalent:

(1) $T(L)$ is a Boolean algebra;

(2) $\operatorname{Min}(L)$ is a compact space.

Proposition 6.8. [2] A bounded distributive lattice $L$ is a Stone lattice if and only if $L$ is conormal and $\operatorname{Min}(L)$ is compact.

Recall that the set $B(L)$ of complemented elements in bounded distributive lattice $L$ is a Boolean algebra. The complement of an element $e \in B(L)$ is denoted by $\neg e$. Let $u: L \rightarrow L^{\prime}$ be a morphism of bounded distributive lattices. We say that the complemented elements of $L$ can be lifted along $u$ if for each $f \in B\left(L^{\prime}\right)$ there exists $e \in B(L)$ such that $u(e)=f$.

Theorem 6.9. If $L$ is a bounded distributive lattice, then the following properties are equivalent:

(1) L is a Stone lattice; 
(2) $L$ is conormal and $T(L)$ is a Boolean algebra;

(3) $T(L)$ is a Boolean algebra and for each $\wedge$-closed subset $S$ of $L$, the complemented elements of $L$ can be lifted along $\pi_{S}: L \rightarrow L / S$;

(4) $T(L)$ is a Boolean algebra and the complemented elements of $L$ can be lifted along $\pi: L \rightarrow$ $T(L)$.

Proof. The equivalence of (1) and (2) follows from Propositions 6.7 and 6.8.

$(1) \Rightarrow(3)$ From the equivalence of $(1)$ and (2) follows that $T(L)$ is a Boolean algebra. Let $S$ be a $\wedge$-closed subset of $L$ and $\pi_{S}: L \rightarrow L / S$ the canonical morphism associated with $S$. One can suppose that $S$ is a filter of $L$. Assume that $x$ is an element of $L$ such that $x / S \in B(L / S)$, so there exists $y \in L$ such that $x / S \vee y / S=1 / S$ and $x / S \wedge y / S=0 / S$. From $(x \vee y) / S=1 / S$ we get $x \vee y \in S$ and $(x \wedge y) / S=0 / S$ implies that $x \wedge y \wedge t=0$, for some $t \in S$. Since $L$ is a Stone lattice it follows that $x^{\perp}=(e]$, for some complemented element $e$ of $L$. Then $e \wedge x=0$, so $x \leq \neg e$, therefore $x / S \leq \neg e / S$. From $x \wedge y \wedge t=0$ we obtain $y \wedge t \in x^{\perp}$, so $y \wedge t \leq e$. We observe that $t / S=1$, because $t \in S$. Thus $y / S=y / S \wedge t / S \leq e / S$, hence $\neg e / S \wedge y / S=0 / S$. Then the following equalities hold:

$$
\neg e / S=\neg e / S \wedge(x / S \vee y / S)=(\neg e / S \wedge x / S) \vee(\neg e / S \wedge y / S)=\neg e / S \wedge x / S=x / S .
$$

Thus $\neg e \in B(L)$ and $\pi_{S}(\neg e)=\neg e / S=x / S$, so the complemented elements of $L$ can be lifted along $\pi_{S}$.

(3) $\Rightarrow$ (4) Obviously.

$(4) \Rightarrow(1)$ Let $x$ be an arbitrary element of $L$. Since $T(L)$ is a Boolean algebra and the complemented elements of $L$ can be lifted along $\pi: L \rightarrow T(L)$, there exists $e \in B(L)$ such that $\pi(e)=x / D$. Thus $e / S=x / S$, so there exists $t \in D$ such that $e \wedge t=x \wedge t$.

We shall prove that $x^{\perp}=(\neg e]$. From $\neg e \wedge x \wedge t=\neg e \wedge e \wedge t=0$ we get $\neg e \wedge x \in t^{\perp}=\{0\}$, hence $\neg e \wedge x=0$. Then $\neg e \in x^{\perp}$ so the inclusion $(\neg e] \subseteq x^{\perp}$ is established. In order to show that $x^{\perp} \subseteq(\neg e]$ assume $z \in x^{\perp}$, i.e. $z \wedge x=0$. It follows that $z \wedge e \wedge t=z \wedge x \wedge t=0$, so $z \wedge e \in t^{\perp}=\{0\}$, hence $z \leq \neg e$. Therefore we get $x^{\perp} \subseteq(\neg e]$, so $x^{\perp}=(\neg e]$. We conclude that $L$ is a Stone lattice.

\section{Conclusion}

In this paper, the relation betwee the lattices of fractions to flat lattice morphisms, patch and flat topologies on the spectra of bounded distributive lattices, conormal and Stone lattices, etc is investigated.

The flat morphisms of $\mathbf{D}_{01}$ in terms of the residuation operation existing in the frames of lattice ideals is defined. We studied how the lattices of fractions preserve the flatness property of morphisms. Two characterization theorems of flat and patch topologies are proved. The lattices of fractions are used for obtaining new characterizations of conormal and Stone lattices.

\section{References}

[1] M. Aghajani, A. Tarizadeh, Characterization of Gelfand rings, specially of clean rings and their dual rings, Results in Mathematics, 75(3) (2020), 125. 
[2] H. Al-Ezeh, On $\sigma-$ ideals of conormal lattices, Commentarii mathematici Universitatis Sancti Pauli, 40(1) (1991), 7-13.

[3] H. Al-Ezeh, Further results on reticulated rings, Acta Mathematica Hungarica, 60 (1-2) (1992), $1-6$.

[4] M.F. Atiyah, I.G. MacDonald, Introduction to commutative algebra, Addison Wesley Publishing Company, 1969.

[5] R. Balbes, P. Dwinger, Distributive lattices, University of Missouri Press, 1974.

[6] B. Banaschewski, Gelfand and exchange rings: Their spectra in pointfree topology, The Arabian Journal for Science and Engineering, 25(2C) (2000), 3-22.

[7] G. Birkhoff, Lattice theory, 3rd ed., AMS Colloquium Publications, 25 (1967).

[8] N. Bourbaki, Algébre commutative, Chapitres 1-4, Springer-Verlag Berlin Heidelberg, 2006.

[9] A. Brezuleanu, Sur les schemas of lattices, Revue Roumaine des Mathematiques Pures et Appliquees, 14 (1969), 949-954.

[10] A. Brezuleanu, R. Diaconescu, Sur la duale de la categorie des treillis, Revue Roumaine des Mathematiques Pures et Appliquees, 14 (1969), 311-323.

[11] D. Cheptea, G. Georgescu, C. Mureşan, Boolean lifting properties for bounded distributive lattices, Scientific Annals of Computer Science, 25 (2015), 29-67.

[12] R. Cignoli, The lattice of global sections of sheaves of chains over Boolean spaces, Algebra Universalis, 8 (1978), 357-373.

[13] W.H. Cornish, Normal lattices, Journal of the Australian Mathematical Society, 14 (1972), 200-215.

[14] W.H. Cornish, O-ideals, congruences and sheaf representations of distributive lattices, Revue Roumaine des Mathematiques Pures et Appliquees, 22(8) (1977), 1059-1067.

[15] A.J. De Jong et al., Stacks project, see http://stacs.math.columbia.edu.

[16] M. Dickmann, N. Schwartz, M. Tressl, Spectral spaces, Cambridge University Press, 2019.

[17] D. Doobs, M. Fontana, I. Papick, On the flat spectral topology, Rendiconti Mathematics, 1(4) (1981), 559-578.

[18] N. Galatos, P. Jipsen, T. Kowalski, H. Ono, Residuated lattices: An algebraic glimpse at structural logics, Studies in Logic and The Foundation of Mathematics, 151, Elsevier, 2007.

[19] G. Georgescu, F-multipliers and the localization of bounded distributive lattices, Algebra Universalis, 21 (1985), 181-197.

[20] G. Georgescu, F-multipliers and the localization of bounded distributive lattices II, Mathematical Logic Quarterly, 37 (1991), 203-300.

[21] G. Georgescu, Flat topology on the spectra of quantales, Fuzzy Sets Systems, 2020, doi:10.1016/j.fss.2020.08.009. 
[22] G. Georgescu, I. Voiculescu, Isomorphic sheaf representations of normal lattices, Journal of Pure and Applied Algebra, 45 (1987), 213-223.

[23] A.W. Hager, C.M. Kimber, W.Wm. McGovern, Clean unital $\ell$-groups, Mathematica Slovaca, 63 (2013), 979-992.

[24] M. Hochster, Prime ideals structures in commutative rings, Transactions of the American Mathematical Society, 142 (1969), 43-60.

[25] N.A. Immormino, Some notes on clean rings, Bowling Green State University, 2012.

[26] P. Jipsen, Generalization of Boolean products for lattice-ordered algebras, Annals of Pure and Applied Logic, 161 (2009), 224-234.

[27] P.T. Johnstone, Stone spaces, Cambridge University Press, 1982.

[28] T.Y. Lam, Lectures on modules and rings, Graduate Texts in Mathematics, 189, Springer Verlag, 1999.

[29] W.Wm. McGovern, Neat rings, Journal of Pure and Applied Algebra, 205 (2006), 243-265.

[30] W.K. Nicholson, Lifting idempotents and exchange rings, Transactions of the American Mathematical Society, 229 (1977), 268-278.

[31] Y.S. Pawar, Characterization of normal lattices, Indian Journal of Pure and Applied Mathematics 24(11) (1993), 651-656.

[32] G. Picavet, Ultrafiltres sur une espace spectral-Anneaux de Baer-Anneaux a spectre minimal compact, Mathematica Scandinavica, 46 (1980), 23-53.

[33] H. Simmons, Reticulated rings, Journal of Algebra, 66 (1980), 169-192.

[34] T.P. Speed, Spaces of ideals of distributive lattices II: Minimal prime ideals, Journal of the Australian Mathematical Society, 18(1) (2009), 72.

[35] A. Tarizadeh, Zariski compactness of minimal spectrum and flat compactness of maximal spectum, Journal of Algebra and Its Applications, 18(11) (2018), doi.org/10.1142/S0219498819502025.

[36] A. Tarizadeh, Flat topology and its dual aspects, Communications in Algebra, 47(1) (2019), 105-205.

[37] A. Tarizadeh, Structure theory of P.P. rings and their generalizations, ArXiv:2001.10419v4, Jun 2020.

[38] A. Tarizadeh, M. Aghajani, On purely-prime ideals with applications, ArXiv: 2001.04823, January 2020.

[39] A. Tarizadeh, M. Aghajani, Structural results on harmonic rings and lessened rings, ArXiv:2006.14036v1, Jun 2020. 